\title{
miR-203 regulates the proliferation, apoptosis and cell cycle progression of pancreatic cancer cells by targeting Survivin
}

\author{
DONG XU ${ }^{1}$, QINGGUANG WANG ${ }^{1}$, YONG $\mathrm{AN}^{2}$ and LIJIAN XU ${ }^{1}$ \\ ${ }^{1}$ Department of General Surgery, The Second Affiliated Hospital of Nanjing Medical University, Nanjing, \\ Jiangsu 210018; ${ }^{2}$ Laboratory of General Surgery, The First Affiliated Hospital of Nanjing Medical University, \\ Nanjing, Jiangsu 210009, P.R. China
}

Received January 24, 2013; Accepted May 23, 2013

DOI: $10.3892 / \mathrm{mmr} .2013 .1504$

\begin{abstract}
MicroRNAs have emerged as crucial regulators of tumorigenesis. However, the mechanism by which miR-203 is involved in the pathogenesis of pancreatic cancer (PC) remains elusive. In the present study, PC cell lines were used as an experimental model to investigate the expression and functional role of miR-203 in PC. miR-203 mimic virus, miRNA negative control virus and Survivin shRNA virus were transfected into the PC cell line, CFPAC-1. mRNA and protein levels of Survivin were detected using qPCR and western blot analysis. Proliferation, apoptosis and cell cycle profiles were detected by an MTT assay and flow cytometry. Female BALB/cA-nu nude mice were used to validate the role of miR-203 in vivo. The protein levels of Survivin were found to negatively correlate with miR-203 levels in four PC cell lines. A luciferase assay revealed that Survivin was a direct target of miR-203. Transfection with miR-203 mimic inhibited CFPAC-1 cell proliferation and induced apoptosis and $G_{1}$ phase cell cycle arrest, similar to knockdown of Survivin. In the in vivo nude mouse model, the downregulation of Survivin by knockdown of Survivin or transfection with miR-203 mimic inhibited tumor growth. Results of the current study indicate that miR-203 regulates the proliferation, apoptosis and cell cycle progression of PC cells by targeting Survivin.
\end{abstract}

\section{Introduction}

Pancreatic cancer (PC) is a highly metastatic malignancy. Despite advanced developments in surgery, radiation therapy and chemotherapy for the treatment of PC, the 5-year survival rate following surgery in $\mathrm{PC}$ patients remains extremely poor

Correspondence to: Dr Lijian Xu, Department of General Surgery, The Second Affiliated Hospital of Nanjing Medical University, 121 Jiangjiayuan Road, Nanjing, Jiangsu 210018, P.R. China E-mail: xulijian185@126.com

Key words: miR-203, Survivin, apoptosis, pancreatic cancer, $\mathrm{G}_{1}$ cell cycle arrest
(15-20\%) (1). Therefore, it is urgent to identify new therapeutic targets or early diagnosis markers for PC.

Survivin (BIRC5) is a member of the inhibitors of apoptosis protein (IAP) family, which includes seven other members, as follows: X-linked inhibitor of apoptosis, cIAP1, cIAP2, NAIP, livin, IAP-like protein 2 and BRUCE (2). Survivin functions to regulate cell division, apoptosis, cellular stress responses and surveillance checkpoints, and its expression is abnormally high in a number of human malignancies, including esophageal, stomach, liver, brain, lung, breast, ovary and hematological cancer (3-5). The overexpression of Survivin is associated with advanced disease, resistance to therapy, reduced survival and induced recurrence (6). By contrast, the downregulation of Survivin may reduce cell proliferation and increase sensitivity to radiotherapy and cytotoxic drugs in various cancer cell lines, including head, neck, thyroid, lung, bladder, cervical and renal cancer (7-11). These observations indicate that Survivin may represent a molecular target for human cancer therapy.

MicroRNAs are a novel class of endogenous single-stranded and non-coding RNA molecules. They are 19-24 nucleotides long and function as gene expression regulators by targeting the 3'-untranslated region (UTR) of mRNA for degradation or translational repression (12). microRNAs have been identified to regulate cell proliferation, apoptosis, migration, invasion and the cell cycle in various cancer cell lines (13). In addition, it was previously reported that the elevated expression of miR-203 is associated with poor survival and may be used as a new prognostic marker for PC $(14,15)$. Notably, while miR-203 is downregulated in hematopoietic malignancies and prostate cancer, it is upregulated in ovarian, bladder and colon cancer (16-20). These studies indicate that the role of miR-203 in tumorigenesis is complex. More recent studies have reported that miR-203 inhibits the proliferation of hepatocellular carcinoma (HCC) and laryngeal cancer cells by targeting Survivin $(21,22)$.

In the present study, PC cell lines were used as an experimental model to investigate the expression and role of miR-203 in PC. In addition, we explored the relationship between miR-203 and Survivin expression and function, and aimed to determine whether miR-203 directly targets Survivin in PC cells to inhibit cancer progression. 


\section{Materials and methods}

Cell culture. The human PC cell lines, SW1990, CFPAC-1, Panc-1 and BxPc-3, were obtained from Shanghai Cell Bank (Shanghai, China) and cultured in Dulbecco's modified Eagle's medium (DMEM) supplemented with $10 \%$ fetal bovine serum (FBS; both Wisent Inc., St-Bruno, QC, Canada), $100 \mu \mathrm{g} / \mathrm{ml}$ streptomycin, $100 \mu \mathrm{g} / \mathrm{ml}$ penicillin and $2 \mathrm{mM}$ glutamine in a humidified chamber at $37^{\circ} \mathrm{C}$ with $5 \% \mathrm{CO}_{2}$.

miRNA and siRNA transfection. miRNA and Survivin shRNA virus (shSurvivin) were designed and synthesized by Genepharma (Shanghai, China). CFPAC-1 cells were seeded in $6-\mathrm{cm}$ tissue culture plates at a density of $50 \%$. After $24 \mathrm{~h}$, the cells were transfected with miRNAs or shSurvivin virus using reduced serum medium (OPTI-MEM-I) according to the manufacturer's instructions. At $48 \mathrm{~h}$ post-transfection, the fluorescent index of the cells reached $90 \%$.

$q P C R$. Total RNA was extracted from the cells using TRIzol reagent (Invitrogen Life Technologies, Carlsbad, CA, USA), and Primescript RT reagent (Takara Bio, Inc., Shiga, Japan) was used to synthesize cDNA. qPCR was performed with a 7500 Real-Time-PCR System (Applied Biosystems, Foster City, CA, USA) using the following primers: miR-203 forward, 5'-GTCGTTACCAGTGCAGGGTCCGAGG TATTCGCACTGGATACGACCTAGT-3' and reverse, 5'-GCCCGTGAAATGTTTAGGACCAC-3'; U6 forward, 5'-ATTGGAACGATACAGAGAAGATT-3' and reverse, 5'-GGAACGCTTCACGAATTTG-3'; Survivin forward, 5'-AGGACCACCGCATCTCTACATTC-3' and reverse, 5'-CCTTGAAGCAGAAGAAACACTGGG-3'; and GAPDH forward, 5'-TCACCCACACTGTGCCCATCTACGA-3' and reverse, 5'-CAGCGGAACCGCTCATTGCCAATGG-3'. GAPDH mRNA and U6 were used as internal controls for determining the relative expression levels of Survivin mRNA and miR-203, respectively. The comparative $\Delta \Delta \mathrm{Ct}$ method was used to calculate the relative expression levels of mRNA and miRNA, and the fold-changes were analyzed by $2^{-\Delta \Delta C t}$.

Western blot analysis. Total protein was extracted from the cells using RIPA buffer containing $1 \%$ phenylmethylsulfonyl fluoride (PMSF) and the protein concentration was estimated with a BCA kit (Nanjing KeyGen Biotech. Co., Ltd., Nanjing, China). Protein was resolved by $12 \%$ sodium dodecyl sulfate polyacrylamide gel electrophoresis and transferred to polyvinylidene difluoride membranes. The membranes were blocked in Tris-buffered saline with $5 \%$ non-fat dry milk at $4^{\circ} \mathrm{C}$ for $12 \mathrm{~h}$ and subsequently incubated with rabbit polyclonal anti-Survivin antibody (Abcam, Cambridge, MA, USA) or mouse monoclonal anti-GAPDH antibody (Beyotime, Jiangsu, China) at $4^{\circ} \mathrm{C}$ for $12 \mathrm{~h}$, followed by incubation with horseradish peroxidase-conjugated goat anti-rabbit or goat anti-mouse secondary antibody (Beyotime, Jiangsu, China) for $2 \mathrm{~h}$ at room temperature. Membranes were developed using an ECL kit (Pierce Biotechnology, Inc., Rockford, IL, USA) and exposed onto X-ray films to visualize the images. GAPDH served as a loading control.

Dual luciferase reporter assay. Four oligos corresponding to the 3'UTR of Survivin were synthesized as follows: wild type, 5'-CTAGATAAAAAGCCTGTCATTTCAAACACTGC TGTGGACGGCCGG-3' and 5'-CCGTCCACAGCA GTGTTTGAAATGACAGGCTTTTTAT-3'; and mutant, 5'-CTAGATAAAAAGCCTGTCGCACCA AACACTGCTGTGGACGGCCGG-3' and 5'-CCGTCCA CAGCAGTGTTTGGTGCGACAGGCTTTTTAT-3. The oligos were cloned into the $\mathrm{XbaI}$ site of the pGL3 luciferase reporter gene (Promega Corporation, Madison, WI, USA) to generate pGL3-Survivin-3'UTR and pGL3-Survivin-3'UTR-mut vectors. CFPAC-1 cells were cultured in 24-well tissue culture plates and co-transfected with $200 \mathrm{ng}$ pGL3-Survivin or pGL3-Survivin-mut and $20 \mathrm{ng}$ pRL-SV40 (Promega Corporation) containing Renilla luciferase and $20 \mathrm{pmol} 203 \mathrm{M}$ or $203 \mathrm{NC}$. At $48 \mathrm{~h}$ post-transfection, cells were collected and a Dual-Luciferase Reporter assay kit (Promega Corporation) was used to detect luciferase activity according to the manufacturer's instructions. All experiments were performed in triplicate.

Cell proliferation, apoptosis and cell cycle analysis. An MTT kit (Nanjing KeyGen Biotech. Co., Ltd.) was used to determine cell proliferation. The cells were seeded in 96-well tissue culture plates (Costar; Corning Inc., Acton, MA, USA) at a density of $2 \times 10^{3}$ cells/well $24 \mathrm{~h}$ prior to transfection with miRNA or siRNA, and then replaced with 10\% FBS-DMEM $6 \mathrm{~h}$ post-transfection and incubated for $48 \mathrm{~h}$. MTT assays were performed daily for 6 days as described previously (23). Cell cycle and apoptosis were detected by flow cytometry (BD Biosciences, Franklin Lakes, NJ, USA) as described previously (23). All experiments were performed in triplicate.

Xenografts. A total of 16 four-week-old female nude BALB/cA-nu (nu/nu) mice were obtained from the Shanghai Experimental Animal Center (Chinese Academy of Sciences, Shanghai, China) and randomly divided into four groups. CFPAC-1, CFPAC-1-203NC, CFPAC-1-203M and CFPAC-1-shSurvivin cells were administered via a unilateral subcutaneous injection into the flanks of the mice $\left(10^{6}\right.$ cells $/ 100 \mu \mathrm{l} /$ flank). Tumors were measured using vernier calipers every 5 days and the tumor volume was calculated using the following formula: (width ${ }^{2} \mathrm{x}$ length)/2. The mice were then sacrificed after 30 days.

Statistical analysis. All data are presented as the mean \pm SD. A Student's t-test was used to analyze the differences between groups. Statistical analysis was performed with SPSS software (version 16.0; SPSS, Inc., Chicago, IL, USA). P $<0.05$ was considered to indicate a statistically significant difference.

\section{Results}

hsa-miR-203 inhibits Survivin protein expression in PC cells. Firstly, hsa-miR-203 and Survivin levels were examined in four PC cell lines. qPCR revealed that miR-203 expression levels were highest in BxPc-3 cells and lowest in CFPAC-1 cells (Fig. 1A). Western blot analysis revealed that Survivin protein levels were highest in CFPAC-1 cells and lowest in BxPc-3 cells (Fig. 1B). These results indicate that Survivin protein and hsa-miR-203 levels are negatively correlated in PC cells. 


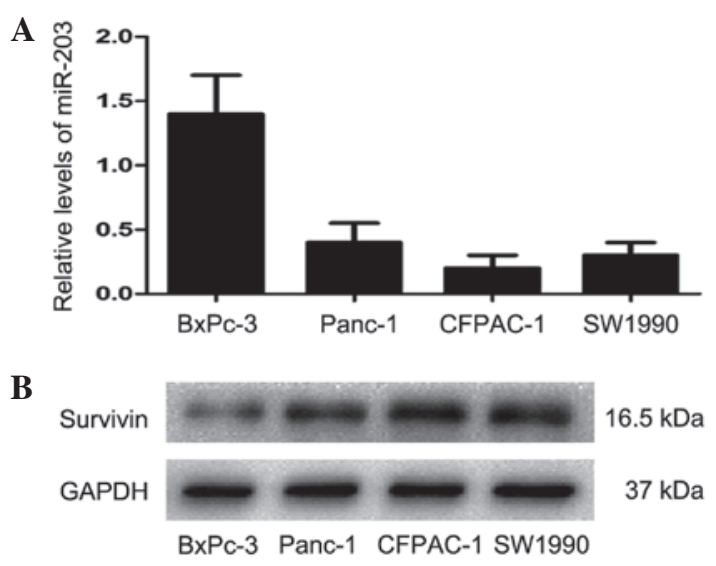

Figure 1. Relative expression levels of miR-203 and Survivin protein in four PC cell lines. (A) miR-203 levels were detected by qPCR and normalized against U6. (B) Western blot analysis of Survivin protein levels. Representative blots from three independent experiments are shown. GAPDH was used as a loading control.
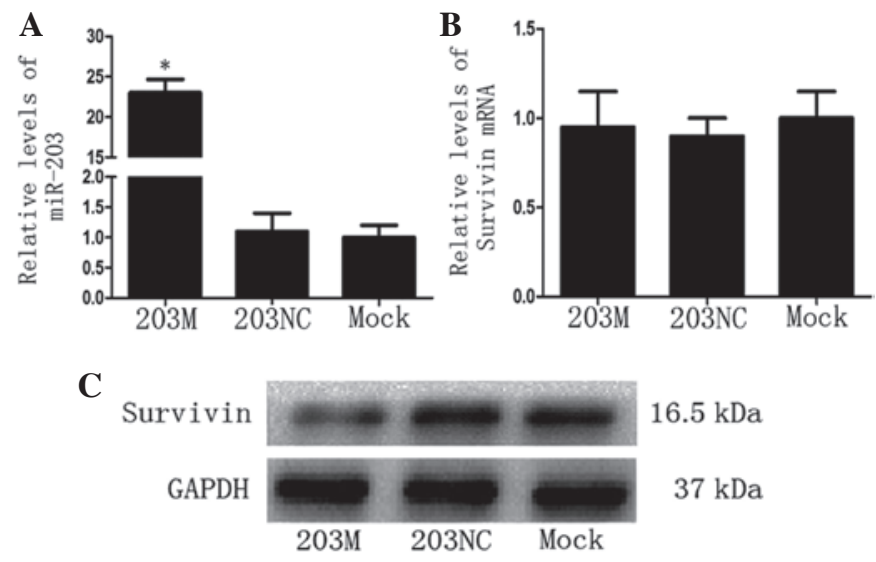

Figure 2. miR-203 inhibits Survivin protein expression. (A) miR-203 levels were detected by qPCR at $48 \mathrm{~h}$ post-transfection in the 203M, 203NC (negative control) and mock (blank control) groups and were normalized against U6. ${ }^{*} \mathrm{P}<0.05$ vs. the $203 \mathrm{NC}$ group. (B) Survivin mRNA levels were detected by q-PCR at $48 \mathrm{~h}$ post-transfection in the 203M, 203NC and mock groups and were normalized to GAPDH mRNA. (C) Western blot analysis of survivin protein levels. Representative blots from three independent experiments are shown. GAPDH was used as a loading control.

Since Survivin protein levels were high in CFPAC-1 cells, the 203M miRNA virus (miR-203 mimic) was transfected into CFPAC-1 cells and Survivin mRNA and protein levels were detected. As predicted, miR-203 levels increased significantly in the $203 \mathrm{M}$ group $(\mathrm{P}<0.05$ vs. the $203 \mathrm{NC}$ group; Fig. $2 \mathrm{~A})$. Notably, compared with the control groups, there was no significant change in Survivin mRNA levels in the 203M group (Fig. 2B); however, Survivin protein levels decreased significantly in the 203M group (Fig. 2C). These results indicate that miR-203 inhibits Survivin expression post-transcriptionally.

Survivin is a direct target gene of miR-203 in PC cells. To confirm that Survivin is a direct target gene of miR-203 in PC cells, TargetScan (http://www.targetscan.org) was used to predict the 3'UTR of Survivin and the binding site of miR-203 (Fig. 3A). Based on this prediction, pGL3-Survivin-3'UTR and pGL3-Survivin-3'UTR-mut
A

$$
\begin{aligned}
& \text { Position 1137-1144 of Survivin 3' UTR } \\
& \text { hsa-miR-203 5' ... AAAUAAAAAGCCUGUCAUUUCAA. . . } \\
& \text { 3' GAUCACCAGGAUUUGUAAAGU }
\end{aligned}
$$

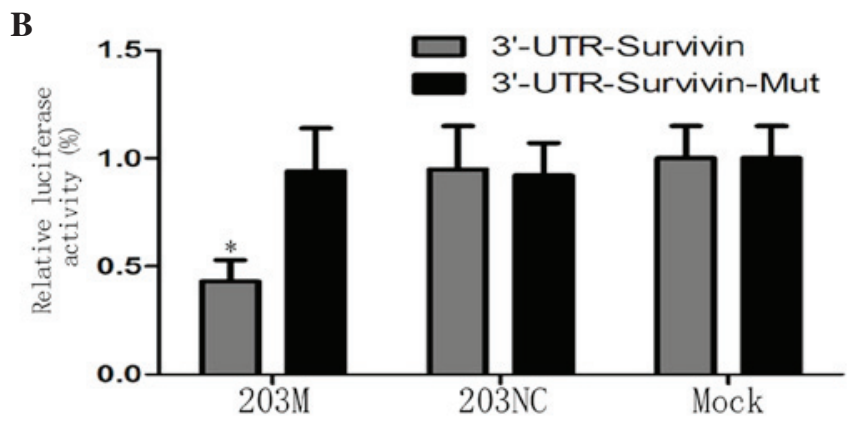

Figure 3. Survivin is a direct target of miR-203 in CFPAC-1 cells (A) TargetScan was used to predict the binding site of miR-203 in the 3'UTR region of Survivin. (B) Transfecting CFPAC-1 cells with pGL3-Survivin vector together with $203 \mathrm{M}$ led to significantly reduced luciferase activity. The data were normalized against the ratio of Renilla luciferase activities. ${ }^{*} \mathrm{P}<0.05$ vs. the $203 \mathrm{NC}$ group. 3'UTR, 3'-untranslated region.

vectors were constructed as a luciferase reporter and control, respectively, and transfected into CFPAC-1 cells. The luciferase assay revealed that luciferase activity was decreased by $\sim 51 \%$ in the $203 \mathrm{M}$ group compared with the controls $(\mathrm{P}<0.05$; Fig. 3B). These results indicate that miR-203 directly targets Survivin via the binding site in its $3^{\prime} \mathrm{UTR}$ region.

hsa-miR-203 inhibits the proliferation and promotes the apoptosis of CFPAC-1 cells. To characterize the role of hsa-miR-203 in PC cells, hsa-miR-203 mimic 203M was transfected into CFPAC-1 cells. Cell proliferation was observed to be significantly inhibited after day 4 when compared with the control ( $\mathrm{P}<0.05$; Fig. 4A). Next, flow cytometry was employed to detect cell cycle progression and apoptosis. CFPAC-1 cells transfected with $203 \mathrm{M}$ revealed a reduced $\mathrm{G}_{2}+\mathrm{S}$ phase compared with the control (41.6 \pm 5.7 vs. $64.7 \pm 5.9 \%$, respectively; $\mathrm{P}<0.05$ ), but exhibited increased $\mathrm{G}_{1}$ phase cell cycle arrest compared with the control $(58.4 \pm 5.3$ vs. $35.3 \pm 4.2 \%$, respectively; $\mathrm{P}<0.05$; Fig. 4B). In addition, the rate of apoptosis was higher in CFPAC- 1 cells transfected with $203 \mathrm{M}$ than in the controls ( $12.2 \pm 2.1$ vs. $5.1 \pm 1.3 \%$, respectively; $\mathrm{P}<0.05$; Fig. 4C). Taken together, these results indicate that miR-203 inhibits the proliferation of CFPAC-1 cells via the induction of $\mathrm{G}_{1}$ phase arrest and apoptosis.

Knockdown of Survivin inhibits the proliferation and promotes the apoptosis of CFPAC-1 cells. Next, the functional implication between miR-203 and Survivin was investigated in PC cells. Following knockdown of Survivin in CFPAC-1 cells by shRNA virus, Survivin mRNA and protein levels were decreased significantly compared with the controls $(\mathrm{P}<0.05$; Fig. 5A). The MTT assay revealed that the proliferation of shSurvivin-transfected cells was decreased after 4 days $(\mathrm{P}<0.05$ vs. control; Fig. $5 \mathrm{C})$. Flow cytometric analysis revealed that the $\mathrm{G}_{2}+\mathrm{S}$ phase was decreased in shSurvivin-transfected cells compared with the controls $(37.3 \pm 4.6$ vs. $67.2 \pm 4.8 \%$, 
A

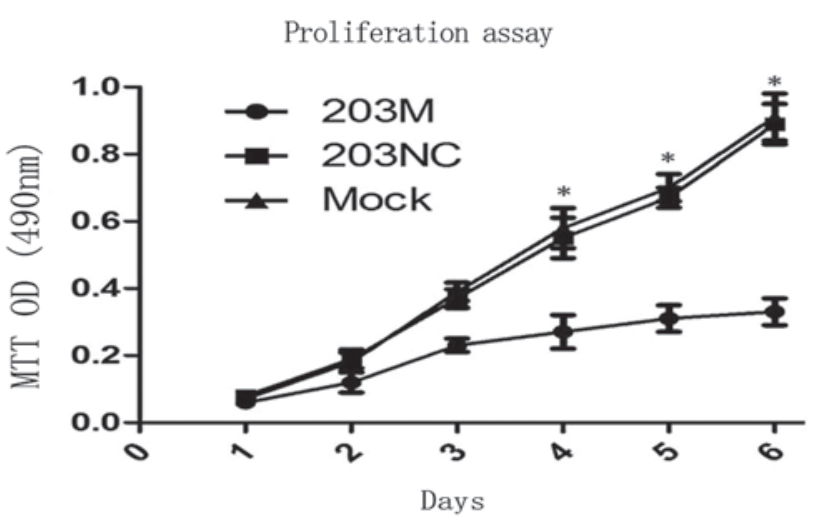

B

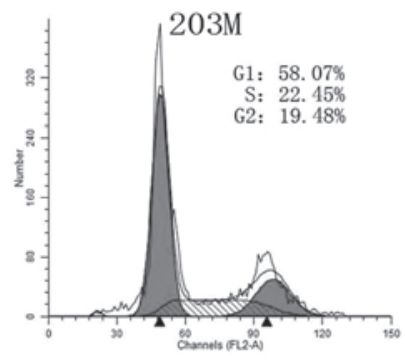

C

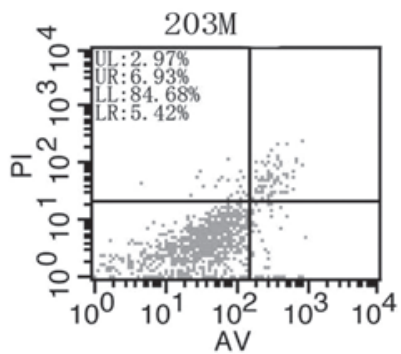

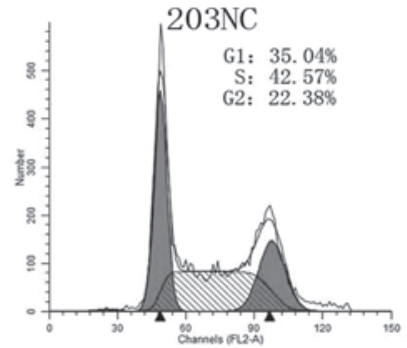
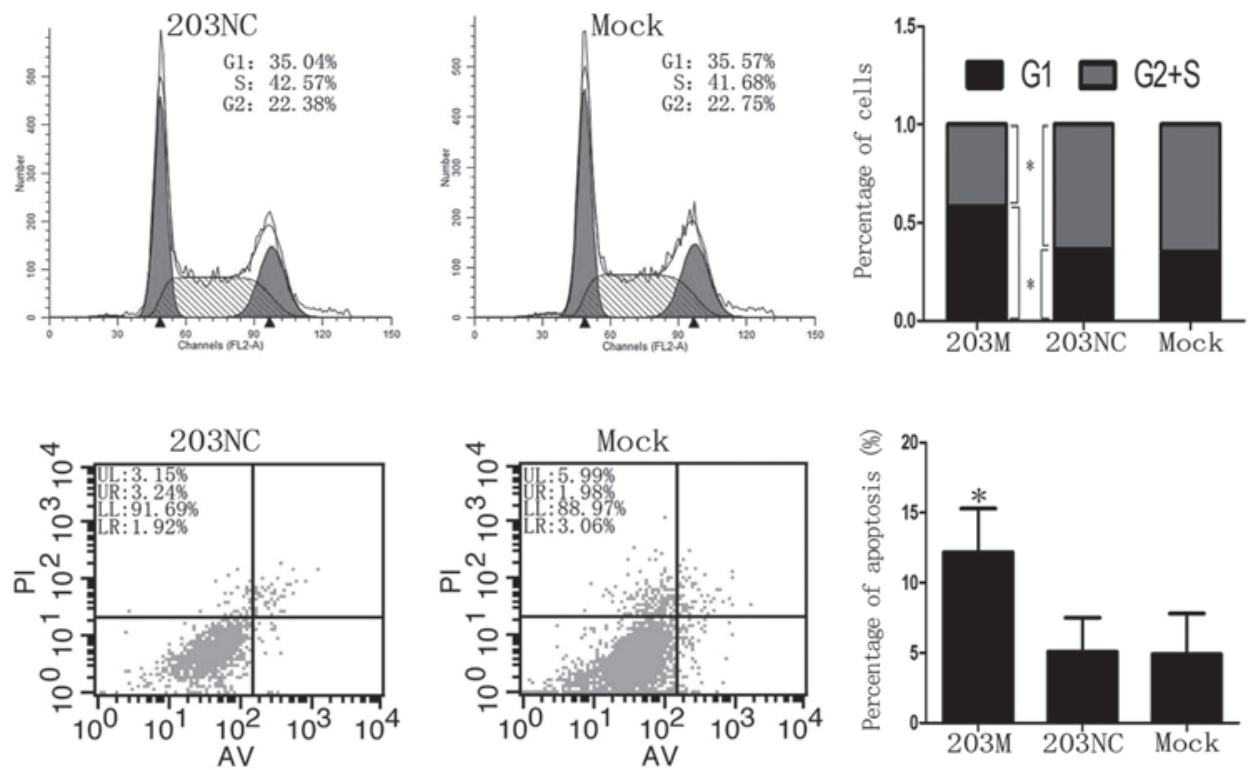

Figure 4. miR-203 inhibits proliferation and induces cell cycle arrest and apoptosis in CFPAC-1 cells. (A) Cell proliferation was detected by an MTT assay. The cell proliferation rate in the 203M group was significantly decreased compared with the control group. (B) Cell cycle profiles were measured by flow cytometry. The 203M group exhibited increased $\mathrm{G}_{1}$ phase and decreased $\mathrm{G}_{2}+\mathrm{S}$ phase compared with the control group. (C) Apoptosis was measured by flow cytometry. The apoptotic rate in the $203 \mathrm{M}$ group was higher than that in the control group. $\mathrm{P}<0.05$ vs. control. AV, Annexin V fluorescein conjugate; PI, propidium iodide.

respectively; $\mathrm{P}<0.05$ ) and the $\mathrm{G}_{1}$ phase was increased in shSurvivin-transfected cells compared with the control $(62.7 \pm 5.3$ vs. $32.8 \pm 3.9 \%$, respectively; $\mathrm{P}<0.05$; Fig. $5 \mathrm{~B}$ ). In addition, the rate of apoptosis in shSurvivin-transfected cells was higher than in the control $(13.4 \pm 4.1$ vs. $4.8 \pm 1.0 \%$, respectively; $\mathrm{P}<0.05$; Fig. 5D). These observations demonstrated that the loss of Survivin produces a similar phenotype as the gain of miR-203 in PC cells, indicating antagonism between Survivin and miR-203.

Downregulation of Survivin inhibits tumor growth in vivo. Finally, the functional antagonism between miR-203 and Survivin in PC was investigated in vivo. Following subcutaneous injection of transfected cells into the flanks of BALB/cA-nu nude mice, the resulting tumors were measured every 5 days for 30 days, followed by euthanasia of the mice. Tumor growth curves revealed that tumors of the shSurvivin group were significantly smaller than those of the control groups after 20 days (Fig. 6A). The tumor sizes in the 203M and shSurvivin groups were smaller than those in the control groups (Fig. 6B). The tumor growth curve demonstrated that tumor growth in the $203 \mathrm{M}$ and shSurvivin groups was slower than that in the control groups (Fig. 6C). These in vivo results are consistent with the in vitro results of this study and further confirm the antagonism between Survivin and miR-203 in PC growth.

\section{Discussion}

In recent years, the aberrant expression of miRNAs has been reported to be implicated in human malignancies $(12,13)$. While miR-203 is downregulated in hematopoietic malignancies and prostate cancer, it is upregulated in ovarian, bladder and colon cancer (16-20). In the present study, miR-203 levels were found to negatively correlate with Survivin levels in PC cells. In addition, in vitro cell proliferation and apoptosis assays, as well as an in vivo xenograft model, demonstrated that miR-203 mimic inhibited the malignant phenotypes of CFPAC-1 cells. Notably, the knockdown of Survivin similarly inhibited the malignant phenotypes of CFPAC-1 cells. A luciferase assay further confirmed that miR-203 inhibited the expression of Survivin by directly targeting its 3'UTR. Survivin is known to promote cancer cell survival and drug 
A

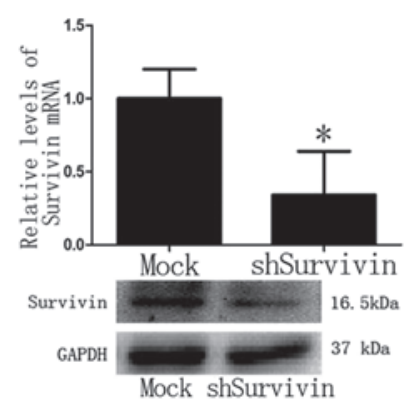

C

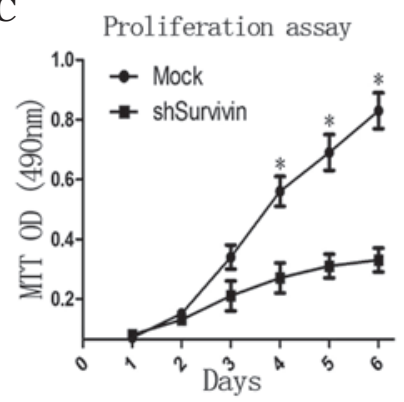

B
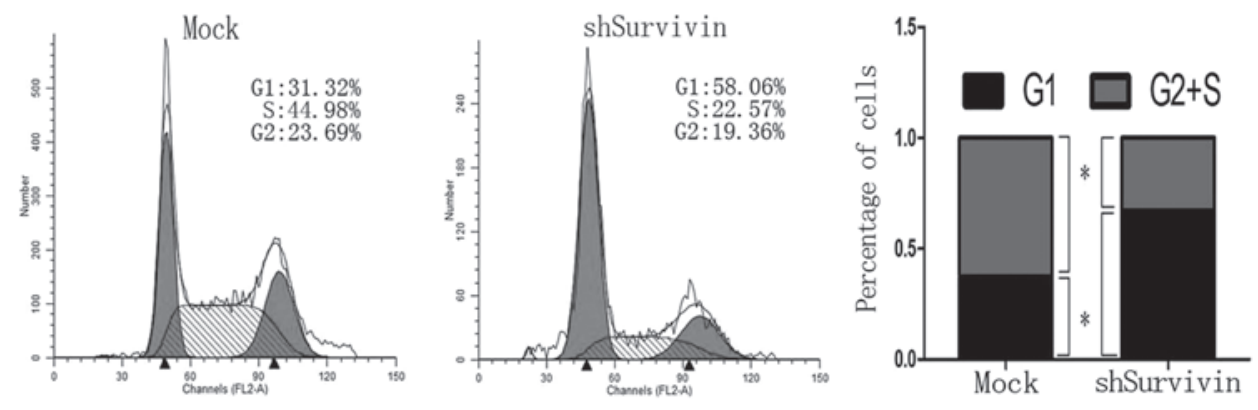

D
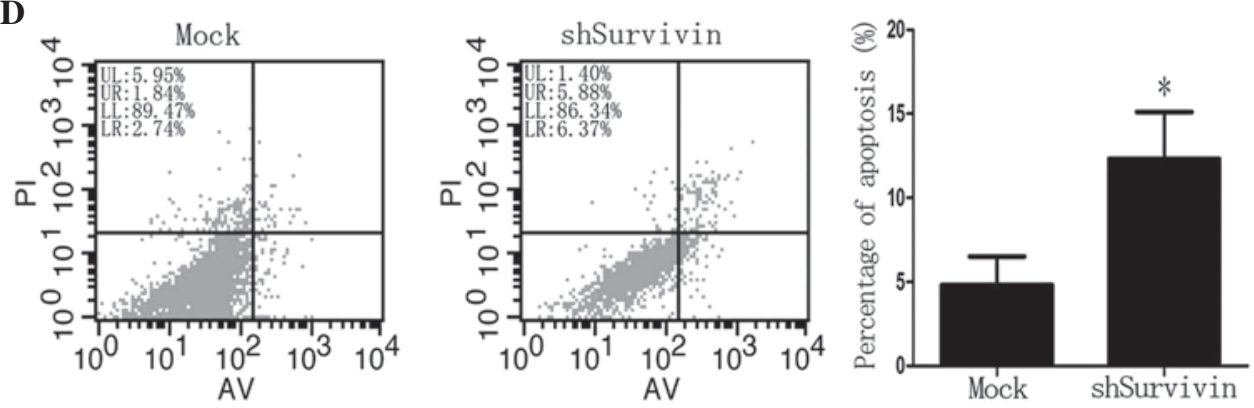

Figure 5. Knockdown of Survivin inhibits proliferation and induces cell cycle arrest and apoptosis in CFPAC-1 cells. (A) q-PCR and western blot analysis for the determination of Survivin mRNA and protein levels in CFPAC-1 cells. GAPDH mRNA and protein levels were used as internal and loading controls. Survivin mRNA and protein levels were significantly decreased in the shSurvivin group compared with the control group. (B) Cell cycle profiles were measured by flow cytometry. The shSurvivin group exhibited an increased $\mathrm{G}_{1}$ phase and decreased $\mathrm{G}_{2}+\mathrm{S}$ phase compared with the control group. (C) Cell proliferation was detected by an MTT assay. The cell proliferation rate in the shSurvivin group was significantly decreased compared with the control group. (D) Apoptosis was measured by flow cytometry. The apoptotic rate in the shSurvivin group was higher than that in the control group. " $\mathrm{P}<0.05$ vs. control. shSurvivin, Survivin shRNA virus; AV, Annexin V fluorescein conjugate; PI, propidium iodide.

A
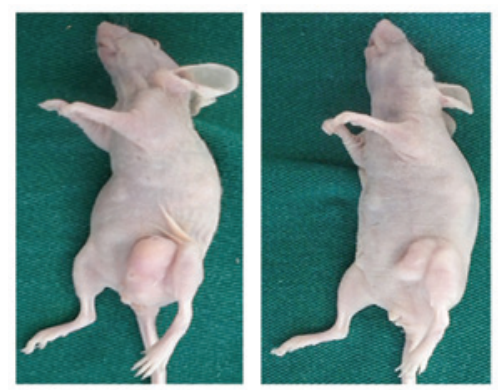

B
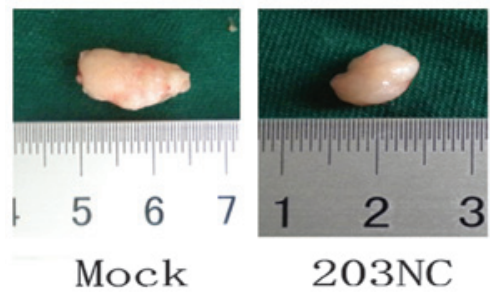

Mock

$203 N C$
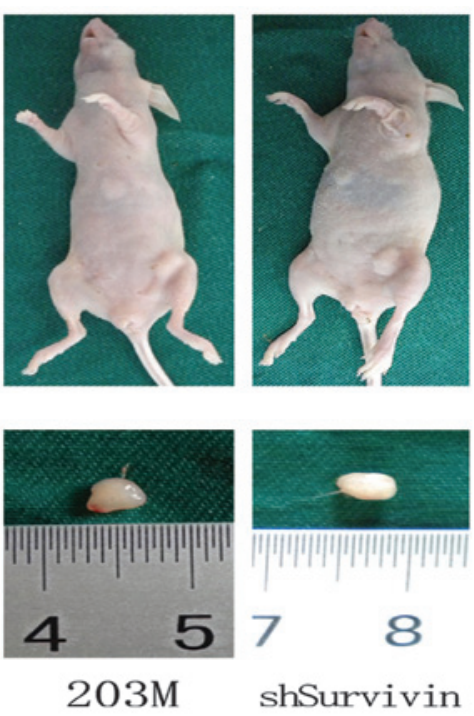

C

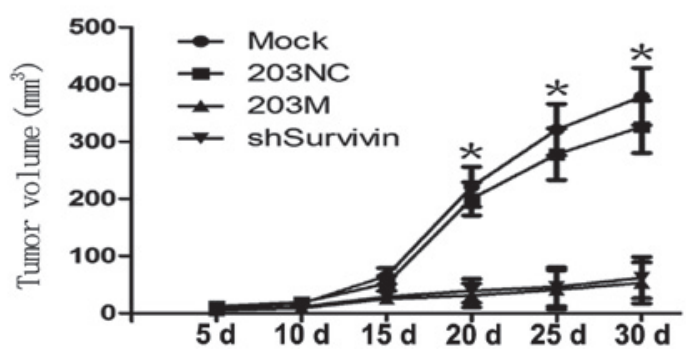

Figure 6. Downregulation of Survivin inhibits tumor growth in vivo. (A) Subcutaneous injection of CFPAC-1, CFPAC-1-203NC, CFPAC-1-203M and CFPAC-1-shSurvivin cells into the flanks of mice $(n=4)$ led to tumor growth. (B) Tumors were removed after 30 days and the tumor size was measured. Tumor sizes in the 203M and shSurvivin groups were smaller than those of the other groups. (C) Tumor growth curve. Following injection with tumor cells, the tumor volume was calculated every five days. ${ }^{*} \mathrm{P}<0.05$ vs. the $203 \mathrm{NC}$ group. shSurvivin, Survivin shRNA virus. 
resistance (6). It is reasonable to hypothesize that miR-203 suppresses the expression of Survivin, leading to its loss of oncogenic function. This prediction is consistent with the downreguation of miR-203 and overexpression of Survivin in PC and indicates that miR-203 is an anti-oncomir, at least in $\mathrm{PC}$. These observations are also consistent with previous results in HCC and laryngeal cancer cells $(21,22)$.

miR-203 was demonstrated to be overexpressed in pancreatic adenocarcinoma samples with advanced disease, and indicated a shorter survival time or poorer prognosis for patients who underwent pancreatectomy $(14,15)$. The same outcome was demonstrated in colon cancer (20). These results indicate that miR-203 functions as an oncomir. By contrast, miR-203 has also been reported to directly target oncogenes, including ABL1, Bcl-w, Runx2, Scr, AKT2 and DNp63, functioning as a tumor-suppressor in esophageal, gastric, hepatocellular, bladder, prostate and colorectal cancer and hematological malignancies (16,23-27). Therefore, the function of miR-203 in different tissues is complex and further studies are required to understand its underlying mechanisms.

In conclusion, in the present study, miR-203 was demonstrated to inhibit the proliferation and induce the apoptosis of PC cells. In addition, Survivin was identified as a direct target of miR-203. These results indicate that miR-203 functions as an anti-oncomir in PC and represents a potential molecular target for PC therapy.

\section{Acknowledgements}

The present study was approved by the ethics committee of Nanjing Medical University, Nanjing, Jiangsu, PR China. (No. NJMU-ERLAUA-20120901). The authors thank Medjaden Bioscience Limited for assistance in the preparation of this manuscript.

\section{References}

1. Liu BB and Wang WH: Survivin and pancreatic cancer. World J Clin Oncol 2: 164-168, 2011.

2. Kelly RJ, Lopez-Chavez A, Citrin D, Janik JE and Morris JC: Impacting tumor cell-fate by targeting the inhibitor of apoptosis protein survivin. Mol Cancer 10: 35, 2011.

3. Yamamoto H, Ngan CY and Monden M: Cancer cells survive with survivin. Cancer Sci 99: 1709-1714, 2008.

4. Pennati M, Folini M and Zaffaroni N: Targeting survivin in cancer therapy. Expert Opin Ther Targets 12: 463-476, 2008.

5. Fukuda S and Pelus LM: Survivin, a cancer target with an emerging role in normal adult tissues. Mol Cancer Ther 5: 1087-1098, 2006.

6. Cao C, Mu Y, Hallahan DE and Lu B: XIAP and survivin as therapeutic targets for radiation sensitization in preclinical models of lung cancer. Oncogene 23: 7047-7052, 2004.

7. Sharma H, Sen S, Lo Muzio L, Mariggiò A and Singh N: Antisense-mediated downregulation of anti-apoptotic proteins induces apoptosis and sensitizes head and neck squamous cell carcinoma cells to chemotherapy. Cancer Biol Ther 4: 720-727, 2005.

8. Du ZX, Zhang HY, Gao da X, Wang HQ, Li YJ and Liu GL: Antisurvivin oligonucleotides inhibit growth and induce apoptosis in human medullary thyroid carcinoma cells. Exp Mol Med 38: 230-240, 2006.
9. Fuessel S, Kueppers B, Ning S, Kotzsch M, Kraemer K, Schmidt U, Meye A and Wirth MP: Systematic in vitro evaluation of survivin directed antisense oligodeoxynucleotides in bladder cancer cells. J Urol 171: 2471-2476, 2004.

10. Zhang Y, Chen ZD, Du CJ, Xu G and Luo W: siRNA targeting survivin inhibits growth and induces apoptosis in human renal clear cell carcinoma 786-O cells. Pathol Res Pract 205: 823-827, 2009.

11. Li QX, Zhao J, Liu JY, Jia LT, Huang HY, Xu YM, Zhang Y, Zhang R, Wang CJ, Yao LB, Chen SY and Yang AG: Survivin stable knockdown by siRNA inhibits tumor cell growth and angiogenesis in breast and cervical cancers. Cancer Biol Ther 5: 860-866, 2006.

12. Hobert O: Gene regulation by transcription factors and microRNAs. Science 319: 1785-1786, 2008.

13. Esquela-Kerscher A and Slack FJ: Oncomirs - microRNAs with a role in cancer. Nat Rev Cancer 6: 259-269, 2006.

14. Greither T, Grochola LF, Udelnow A, Lautenschläger C, Würl P and Taubert H: Elevated expression of microRNAs 155, 203, 210 and 222 in pancreatic tumors is associated with poorer survival. Int J Cancer 126: 73-80, 2010.

15. Ikenaga N, Ohuchida K, Mizumoto K, Yu J, Kayashima T, Sakai H, Fujita H, Nakata K and Tanaka M: MicroRNA-203 expression as a new prognostic marker of pancreatic adenocarcinoma. Ann Surg Oncol 17: 3120-3128, 2010.

16. Bueno MJ, Pérez de Castro I, Gómez de Cedrón M, Santos J, Calin GA, Cigudosa JC, Croce CM, Fernández-Piqueras J and Malumbres M: Genetic and epigenetic silencing of microRNA-203 enhances ABL1 and BCR-ABL1 oncogene expression. Cancer Cell 13: 496-506, 2008.

17. Viticchiè G, Lena AM, Latina A, Formosa A, Gregersen LH, Lund AH, Bernardini S, Mauriello A, Miano R, Spagnoli LG, Knight RA, Candi E and Melino G: MiR-203 controls proliferation, migration and invasive potential of prostate cancer cell lines. Cell Cycle 10: 1121-1131, 2011

18. Iorio MV, Visone R, Di Leva G, Donati V, Petrocca F, Casalini P, Taccioli C, Volinia S, Liu CG, Alder H, Calin GA, Menard S and Croce CM: MicroRNA signatures in human ovarian cancer. Cancer Res 67: 8699-8707, 2007.

19. Gottardo F, Liu CG, Ferracin M, Calin GA, Fassan M, Bassi P, Sevignani C, Byrne D, Negrini M, Pagano F, Gomella LG, Croce CM and Baffa R: Micro-RNA profiling in kidney and bladder cancers. Urol Oncol 25: 387-392, 2007.

20. Schetter AJ, Leung SY, Sohn JJ, Zanetti KA, Bowman ED, Yanaihara N, Yuen ST, Chan TL, Kwong DL, Au GK, Liu CG, Calin GA, Croce CM and Harris CC: MicroRNA expression profiles associated with prognosis and therapeutic outcome in colon adenocarcinoma. JAMA 299: 425-436, 2008.

21. Wei W, Wanjun L, Hui S, Dongyue C, Xinjun Y and Jisheng Z: miR-203 inhibits proliferation of HCC cells by targeting survivin. Cell Biochem Funct 31: 82-85, 2013.

22. Bian K, Fan J, Zhang X, Yang XW, Zhu HY, Wang L, Sun JY, Meng YL, Cui PC, Cheng SY, Zhang J, Zhao J, Yang AG and Zhang R: MicroRNA-203 leads to G1 phase cell cycle arrest in laryngeal carcinoma cells by directly targeting survivin. FEBS Lett 586: 804-809, 2012.

23. Wang F, Xue X, Wei J, An Y, Yao J, Cai H, Wu J, Dai C, Qian Z, $\mathrm{Xu} \mathrm{Z}$ and Miao Y: hsa-miR-520h downregulates ABCG2 in pancreatic cancer cells to inhibit migration, invasion, and side populations. Br J Cancer 103: 567-574, 2010.

24. Bo J, Yang G, Huo K, Jiang H, Zhang L, Liu D and Huang Y: microRNA-203 suppresses bladder cancer development by repressing bcl-w expression. FEBS J 278: 786-792, 2011.

25. Li J, Chen Y, Zhao J, Kong F and Zhang Y: miR-203 reverses chemoresistance in p53-mutated colon cancer cells through downregulation of Akt2 expression. Cancer Lett 304: 52-59, 2011.

26. Saini S, Arora S, Majid S, Shahryari V, Chen Y, Deng G, Yamamura S, Ueno $\mathrm{K}$ and Dahiya R: Curcumin modulates microRNA-203-mediated regulation of the Src-Akt axis in bladder cancer. Cancer Prev Res (Phila) 4: 1698-1709, 2011.

27. Yuan Y, Zeng ZY, Liu XH, Gong DJ, Tao J, Cheng HZ and Huang SD: MicroRNA-203 inhibits cell proliferation by repressing $\Delta \mathrm{Np} 63$ expression in human esophageal squamous cell carcinoma. BMC Cancer 11: 57, 2011. 